University of Texas at El Paso

ScholarWorks@UTEP

$6-2010$

\title{
Spatial Resolution for Processing Seismic Data: Type-2 Methods for Finding the Relevant Granular Structure
}

\author{
Vladik Kreinovich \\ The University of Texas at El Paso, vladik@utep.edu \\ Jaime Nava \\ The University of Texas at El Paso, jenava@miners.utep.edu \\ Rodrigo Romero \\ The University of Texas at El Paso \\ Julio Olaya \\ The University of Texas at El Paso, jolaya@miners.utep.edu \\ Aaron A. Velasco \\ The University of Texas at El Paso, aavelasco@utep.edu \\ Follow this and additional works at: https://scholarworks.utep.edu/cs_techrep

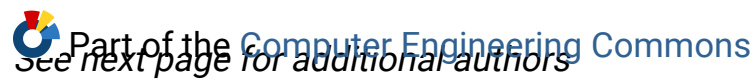 \\ Comments: \\ Technical Report: UTEP-CS-10-12 \\ Published in Proceedings of the IEEE International Conference on Granular Computing GrC'2010, \\ Silicon Valley, USA, August 14-16, 2010.
}

\section{Recommended Citation}

Kreinovich, Vladik; Nava, Jaime; Romero, Rodrigo; Olaya, Julio; Velasco, Aaron A.; and Miller, Kate C., "Spatial Resolution for Processing Seismic Data: Type-2 Methods for Finding the Relevant Granular Structure" (2010). Departmental Technical Reports (CS). 12.

https://scholarworks.utep.edu/cs_techrep/12

This Article is brought to you for free and open access by the Computer Science at ScholarWorks@UTEP. It has been accepted for inclusion in Departmental Technical Reports (CS) by an authorized administrator of ScholarWorks@UTEP. For more information, please contact Iweber@utep.edu. 
Authors

Vladik Kreinovich, Jaime Nava, Rodrigo Romero, Julio Olaya, Aaron A. Velasco, and Kate C. Miller 


\section{Spatial Resolution for Processing Seismic Data: Type-2 Methods for Finding the Relevant Granular Structure}

\author{
Vladik Kreinovich, Jaime Nava, \\ Rodrigo Romero, Julio Olaya, Aaron Velasco \\ Cyber-ShARE Center \\ University of Texas at El Paso \\ El Paso, TX 79968, USA \\ contactvladik@utep.edu
}

\author{
Kate C. Miller \\ Department of Geology and Geophysics \\ Texas A\&M University \\ College Station, TX 77843, USA \\ kcmiller@tamu.edu
}

\begin{abstract}
One of the main methods of determining the Earth structure is the analysis of the seismic data. Based on the seismic data, we produce a 3-D map describing the density at different locations on different depths. Due to incomplete coverage and measurement uncertainty, this map provides only an approximate description of the actual density distribution. For example, based on the seismic data, it is impossible to distinguish between the densities at two nearby points. In other words, what we actually reconstruct is not a function of 3 variables, but rather values determined on the appropriate spatial granules. Because of this granularity, it is necessary to find the spatial resolution at different locations and at different depths, i.e., in other words, it is necessary to determine the corresponding granules. In this paper, we show how Type-2 methods can help in determining these granules.
\end{abstract}

Keywords-spatial resolution; seismic data; granularity

\section{NEED to Find GRANUlarity (SPATIAL Resolution) of THE RESUlts of SEISMic DATA PROCESSING}

In evaluations of natural resources and in the search for natural resources, it is very important to determine Earth structure. Our civilization greatly depends on the things we extract from the Earth, such as fossil fuels (oil, coal, natural gas), minerals, and water. Our need for these commodities is constantly growing, and because of this growth, they are being exhausted. Even under the best conservation policies, there is (and there will be) a constant need to find new sources of minerals, fuels, and water.

The only sure-proof way to guarantee that there are resources such as minerals at a certain location is to actually drill a borehole and analyze the materials extracted. However, exploration for natural resources using indirect means began in earnest during the first half of the 20th century. The result was the discovery of many large relatively easy to locate resources such as the oil in the Middle East.

However, nowadays, most easy-to-access mineral resources have already been discovered. For example, new oil fields are mainly discovered either at large depths, or under water, or in very remote areas - in short, in the areas where drilling is very expensive. It is therefore desirable to predict the presence of resources as accurately as possible before we invest in drilling.

From previous exploration experiences, we usually have a good idea of what type of structures are symptomatic for a particular region. For example, oil and gas tend to concentrate near the top of natural underground domal structures. So, to be able to distinguish between more promising and less promising locations, it is desirable to determine the structure of the Earth at these locations. To be more precise, we want to know the structure at different depths $z$ at different locations $(x, y)$.

Determination of Earth structure is also very important for assessing earthquake risk. Another vitally important application where the knowledge of the Earth structure is crucial is the assessment of earth hazards. Earthquakes can be very destructive, so it is important to be able to estimate the probability of an earthquake, where one is most likely to occur, and what will be the magnitude of the expected earthquake. Geophysicists have shown that earthquakes result from accumulation of mechanical stress; so if we know the detailed structure of the corresponding Earth locations, we can get a good idea of the corresponding stresses and faults present and the potential for occurrence of an earthquake. From this viewpoint, it is also very important to determine the structure of the Earth.

Data that we can use to determine the Earth structure. In general, to determine the Earth structure, we can use different measurement results that can be obtained without actually drilling the boreholes: e.g., gravity and magnetic measurements, analyzing the travel-times and paths of seismic ways as they propagate through the earth, etc.

Seismic measurements are usually the most informative. Because of the importance and difficulty of the inverse problem, geophysicists would like to use all possible measurement results: gravity, magnetic, seismic data, etc. In this paper, we will concentrate on the measurements which carry the largest amount of information about the Earth 
structure and are, therefore, most important for solving inverse problems.

Some measurements - like gravity and magnetic measurements - describe the overall effect of a large area. These measurements can help us determine the average mass density in the area, or the average concentration of magnetic materials in the area, but they often do not determine the detailed structure of this area. This detailed structure can be determined only from measurements which are narrowly focused on small sub-areas of interest.

The most important of these measurements are usually seismic measurements. Seismic measurements involve the recording of vibrations caused by distant earthquakes, explosions, or mechanical devices. For example, these records are what seismographic stations all over the world still use to detect earthquakes. However, the signal coming from an earthquake carries not only information about the earthquake itself, it also carries the information about the materials along the path from an earthquake to the station: e.g., by measuring the travel-time of a seismic wave, checking how fast the signal came, we can determine the velocity of sound $v$ in these materials. Usually, the velocity of sound increases with increasing density, so, by knowing the velocity of sound at different 3 -D points, we will be able to determine the density of materials at different locations and different depths.

The main problem with the analysis of earthquake data (i.e., passive seismic data) is that earthquakes are rare events, and they mainly occur in a few seismically active belts. Thus, we have a very uneven distribution of sources and receivers that results in a "fuzzy" image of earth structure in many areas.

To get a better understanding of the Earth structure, we must therefore rely on active seismic data - in other words, we must make artificial explosions, place sensors around them, and measure how the resulting seismic waves propagate. The most important information about the seismic wave is the travel-time $t_{i}$, i.e., the time that it takes for the wave to travel from its source to the sensor. to determine the geophysical structure of a region, we measure seismic travel times and reconstruct velocities at different depths from these data. The problem of reconstructing this structure is called the seismic inverse problem; see, e.g., [11].

How seismic inverse problem is solved. First, we discretize the problem: we divide our 3-D spatial region into cells, and we consider the velocity values to be constant within each each cell. The value of the velocity in the cell $j$ will be denoted by $v_{j}$.

Once we know the velocities $v_{j}$ in each cell $j$, we can then determine the paths which seismic waves take. Seismic waves travel along the shortest path - shortest in terms of time. As a result, within each cell, the path is a straight line, and on the border between the two cells with velocities $v$ and $v^{\prime}$, the direction of the path changes in accordance with Snell's law

$$
\frac{\sin (\varphi)}{v}=\frac{\sin \left(\varphi^{\prime}\right)}{v^{\prime}}
$$

where $\varphi$ and $\varphi^{\prime}$ are the angles between the paths and the line orthogonal to the border between the cells. (If this formula requires $\sin \left(\varphi^{\prime}\right)>1$, this means that this wave cannot penetrate into the neighboring cell at all; instead, it bounces back into the original cell with the same angle $\varphi$.)

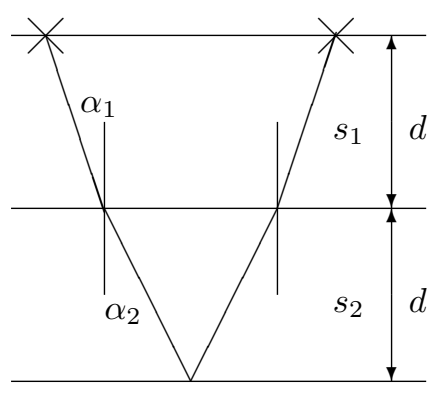

In particular, we can thus determine the paths from the source to each sensor. The travel-time $t_{i}$ along $i$-th path can then be determined as the sum of travel-times in different cells $j$ through which this path passes: $t_{i}=\sum_{j} \frac{\ell_{i j}}{v_{j}}$, where $\ell_{i j}$ denotes the length of the part of $i$-th path within cell $j$.

This formula becomes linear if we replace the original unknowns - velocities $v_{j}-$ by their inverses $s_{j} \stackrel{\text { def }}{=} \frac{1}{v_{j}}$, called slownesses. In terms of slownesses, the formula for the travel-time takes the simpler form $t_{i}=\sum_{j} \ell_{i j} \cdot s_{j}$.

The system is not exactly linear, because the values $\ell_{i j}$ depend on the path and thus, depend on the velocities. To solve this problem, several methods have been proposed. One of the most popular methods, proposed by J. Hole in [6], consists of the following, We start with some initial reasonable values of velocities. Then, we repeat the following two steps until the process converges:

- based on the current values of the slownesses, we find the shortest pathes between sources and sensors and thus, the values $\ell_{i j}$;

- based on the current values $\ell_{i j}$, we solve the above system of linear equations, and get the updated values of slownesses, etc.

Need to find spatial resolution. Based on the seismic data, we produce a 3 -D map describing the velocity $v_{j}$ at different locations on different depths. Due to incomplete coverage and measurement uncertainty, this map provides only an approximate description of the actual velocity distribution. For example, based on the seismic data, it is impossible to distinguish between the densities at two nearby points. In other words, what we actually reconstruct is not a function of 
3 variables, but rather values determined on the appropriate spatial granules. Because of this granularity, it is necessary to find the spatial resolution at different locations and at different depths, i.e., in other words, it is necessary to determine the corresponding granules.

\section{How Granularity (Spatial Resolution) Is DETERMINED Now: SuCCESSES AND LIMITATIONS}

Uncertainty vs. spatial resolution (granularity). Actually, when we reconstruct the velocities in different cells, we have two types of uncertainty (see, e.g., [9], [13]:

- first, the "traditional" uncertainty - the reconstructed value of velocity is, due to measurement inaccuracy and incomplete coverage, only approximately equal to the actual (unknown) velocity value;

- second, the spatial resolution - each measured value represents not the value at a single point, but rather the "average" value over the whole region (granule) that affected the measurement; see, e.g., [12].

Methods of determining traditional uncertainty have been traditionally more developed, and the main ideas of methods for determined spatial resolution comes from these more traditional methods. In view of this, before we describe the existing methods for determining spatial resolution, let us describe the corresponding methods for determining more traditional uncertainty.

How traditional uncertainty is determined: main idea. There exist many techniques for estimating the "traditional" uncertainty; see, e.g., [1], [2], [5], [10] and references therein.

Most of these methods are based on the following idea. Usually, we know the accuracy of different measurements. We therefore

- first, we add, to the measured values of traveltimes, simulated noise of the size of the corresponding measurement errors; these values, due to our selection of noise, could possibly emerge if we simply repeat the same measurements;

- then, we reconstruct the new values of the velocities based on these modified traveltimes; these values come from realistic traveltimes and thus, can occur if we simply repeat the same measurements;

- finally, we compare the resulting velocities with the originally reconstructed ones: the difference between these two reconstructions is a good indication how accurate are these values.

Comment. Since the geophysical models involve a large amount of expert knowledge, it is also necessary to take into account the uncertainty of the expert statements; this is done, e.g., in [2], [3], [4].

How this idea is applied to determine spatial resolution. To determine spatial resolution, we can also simulate noise, the only difference is that this noise should reflect spatial resolution (granularity) and not the inaccuracy of the measurement values. Thus, we arrive at the following method:

- first, we add a perturbation of spatial size $\delta_{0}$ (e.g., sinusoidal) to the reconstructed field $\widetilde{v}(x)$;

- then, we simulate the new traveltimes based on the perturbed values of the velocities;

- finally, we apply the same seismic data processing algorithm to the simulated traveltimes, and reconstruct the new field $\widetilde{v}_{\text {new }}(x)$.

If the perturbations are not visible in $\widetilde{v}_{\text {new }}(x)-\widetilde{v}(x)$, this means that details of spatial size $\delta_{0}$ cannot be reconstructed. If perturbations are

- visible in one area of the map and

- not very clear in the other part of the map,

this means that

- in the first area, we can detect details with spatial resolution $\delta_{0}$ while

- in the second area, the spatial resolution is much lower, and the details of this size are not visible.

In the geosciences, this method is known as a checkerboard method since adding 2-D sinusoidal periodic perturbations makes the map look like a checkerboard.

The use of this method to determine spatial resolution of seismic data processing is described, in detail, in [1], [9], [13], [14]. In particular, in [9], [13], [14], it is proven that the empirically optimal sinusoidal perturbations are actually optimal (under a reasonable formalization of the corresponding optimization problem).

Checkerboard method: main limitation. The main limitation of the checkerboard method is that its running time is several times higher than the time of the original seismic data processing. Indeed,

- in addition to applying the seismic data processing algorithm to the original data,

- we also need to apply the same algorithm to the simulated data - and apply it several times, to make sure that we have reliable results about spatial resolution.

Seismic data processing is usually very time-consuming, often requiring hours and even days of computations on high performance computers. Thus, if we want to compute not only the 3-D maps themselves, but also the spatial resolution of the corresponding maps, the computation time drastically increases - and the whole process slows down.

It is therefore desirable to develop faster techniques for estimating spatial resolution of the corresponding maps, techniques that will not require new processing of simulated seismic data - and will only use the results of the processing the original seismic data.

A similar problem arises for estimating traditional uncertainty. As we have mentioned, the existing methods for determining traditional uncertainty are also based on 
simulating errors and applying the (time-consuming) seismic data processing algorithms to the simulated traveltimes. As a result, the existing methods for determining the traditional uncertainty are also too time-consuming, and there is a similar need to developing faster uncertainty estimation techniques.

Since, as we mentioned, spatial resolution techniques usually emulate techniques for determining traditional uncertainty, let us therefore start with describing the existing techniques for faster

First heuristic idea for estimating uncertainty: ray coverage. In general, each measurement adds information about the affected quantities. The more measurements we perform, the more information we have and thus, the more accurately we can determine the desired quantity.

In particular, for each cell $j$, the value $v_{j}$ affects those traveltime measurements $t_{i}$ for which the corresponding path goes through this cell, i.e., for which $\ell_{i j}>0$. Thus, the more rays pass through the cell, the more accurate the corresponding measurement. The number of such rays called a ray coverage - is indeed reasonably well correlated with uncertainty and can, thus, serve as an estimate for this uncertainty:

- the smaller the ray coverage,

- the larger the uncertainty.

Limitations of ray coverage and the DWS idea. Simply counting the ray does not take into account that some ray barely tough the cell, withe the values $\ell_{i j}$ very small. Clearly, such rays do not add much to the accuracy of determining the velocity $v_{j}$ in the corresponding cell. It is therefore necessary to take into account not only how many rays go through the cell, but also how long are the paths of each ray in each cell. This idea was originally proposed by C. H. Thurber (personal communication, 1986) under the name of the Derivative Weight Sum; it was first published in [17].

As the name implies, instead of simply counting the rays that pass through a given cell $j$, we instead compute the sum of the lengths $D(j)=\sum_{i} \ell_{i j}$. This method have been successfully used in several geophysical problems; see, e.g., [15], [18], [19], [20]. It is indeed better correlated with the actual (simulation-computed) accuracy that the ray coverage.

Comment. The above form of the DWS is based on Hole's code approach, in which the slowness is assumed to be constant within each cell. An alternative approach is assuming that the slowness function is not piece-wise constant but rather piece-wise linear. In other words, we determine the values $s_{j}$ at different points $j=\left(j_{1}, j_{2}, j_{3}\right)$ on a rectangular grid (here $j_{i}$ are assumed to be integers), and we use linear extrapolation to describe the values $s\left(x_{1}, x_{2}, x_{3}\right)$ at arbitrary points $x_{i}=j_{i}+\alpha_{i}$ with $0 \leq \alpha_{i} \leq 1$.
In the 1-D case, linear interpolation takes the simple form $s(x)=\alpha \cdot s_{j+1}+(1-\alpha) \cdot s_{j}$. To get the formula for the 2-D case, we first use linear interpolation to get the values

$$
s\left(x_{1}, j_{2}\right)=\alpha_{1} \cdot s_{j_{1}+1, j_{2}}+\left(1-\alpha_{1}\right) \cdot s_{j_{1}, j_{2}}
$$

and

$$
s\left(x_{1}, j_{2}+1\right)=\alpha_{1} \cdot s_{j_{1}+1, j_{2}+1}+\left(1-\alpha_{1}\right) \cdot s_{j_{1}, j_{2}+1}
$$

and then use 1-D linear interpolation to estimate $s\left(x_{1}, x_{2}\right)$ as

$$
s\left(x_{1}, x_{2}\right)=\alpha_{2} \cdot s\left(x_{1}, j_{2}+1\right)+\left(1-\alpha_{2}\right) \cdot s\left(x_{1}, j_{2}\right),
$$

i.e., substituting the above expressions for $s\left(x_{1}, j_{2}+1\right)$ and $s\left(x_{1}, j_{2}\right)$, the expression

$$
\begin{gathered}
s\left(x_{1}, x_{2}\right)=\alpha_{1} \cdot \alpha_{2} \cdot s_{j_{1}+1, j_{2}+1}+\left(1-\alpha_{1}\right) \cdot \alpha_{2} \cdot s_{j_{1}, j_{2}+1}+ \\
\alpha_{1} \cdot\left(1-\alpha_{2}\right) \cdot s_{j_{1}+1, j_{2}}+\left(1-\alpha_{1}\right) \cdot\left(1-\alpha_{2}\right) \cdot s_{j_{1}, j_{2}} .
\end{gathered}
$$

Similarly, we can go from the 2-D to the 3-D case, resulting in

$$
\begin{gathered}
s\left(x_{1}, x_{2}, x_{3}\right)= \\
\alpha_{3} \cdot s\left(x_{1}, x_{2}, j_{3}+1\right)+\left(1-\alpha_{3}\right) \cdot s\left(x_{1}, x_{2}, j_{3}\right),
\end{gathered}
$$

and

$$
\begin{gathered}
s\left(x_{1}, x_{2}, x_{3}\right)=\alpha_{1} \cdot \alpha_{2} \cdot \alpha_{3} \cdot s_{j_{1}+1, j_{2}+1, j_{3}+1}+ \\
\left(1-\alpha_{1}\right) \cdot \alpha_{2} \cdot \alpha_{3} \cdot s_{j_{1}, j_{2}+1, j_{3}+1}+ \\
\alpha_{1} \cdot\left(1-\alpha_{2}\right) \cdot \alpha_{3} \cdot s_{j_{1}+1, j_{2}, j_{3}+1}+ \\
\left(1-\alpha_{1}\right) \cdot\left(1-\alpha_{2}\right) \cdot \alpha_{3} \cdot s_{j_{1}, j_{2}, j_{3}+1}+ \\
\alpha_{1} \cdot \alpha_{2} \cdot\left(1-\alpha_{3}\right) \cdot s_{j_{1}+1, j_{2}+1, j_{3}}+ \\
\left(1-\alpha_{1}\right) \cdot \alpha_{2} \cdot\left(1-\alpha_{3}\right) \cdot s_{j_{1}, j_{2}+1, j_{3}}+ \\
\alpha_{1} \cdot\left(1-\alpha_{2}\right) \cdot\left(1-\alpha_{3}\right) \cdot s_{j_{1}+1, j_{2}, j_{3}}+ \\
\left(1-\alpha_{1}\right) \cdot\left(1-\alpha_{2}\right) \cdot\left(1-\alpha_{3}\right) \cdot s_{j_{1}, j_{2}, j_{3}} .
\end{gathered}
$$

Under this linear interpolation, in the formula for $t_{i}$, the coefficient at each term $s_{j}$ is no longer $\ell_{i j}$, but rather the integral of the corresponding interpolation coefficient $\omega_{i j}(x)$ over the ray path $\gamma_{i}: \int \omega_{i j}(x) d \gamma_{i}$. Thus, instead of the sum of the lengths, it is reasonable to take the sum of these integrals $D(j)=\sum_{i} \int \omega_{i j}(x) d \gamma_{i}$. This is actually the original form of the DWS.

Angular diversity: a similar approach to spatial resolution. If we have many rays passing through the cell $j$, then we can find the slowness $s_{j}$ in this cell with a high accuracy. However, if all these rays are parallel and close to each other, then all of them provide the information not about this particular cell, but rather about a block of cells following the common path. Thus, in effect, instead of the value $s_{j}$, we get the average value of slowness over several cells - i.e., we get a map with a low spatial resolution. To get 
a good spatial resolution, we must have "angular diversity", rays at different angles passing through the cell $j$.

The measure of such diversity called ray density tensor was proposed in [8]; see also [7], [16], [21]. In this measure, for each cell $j$, we form a $3 \times 3$ tensor (= matrix)

$$
R_{a b}(j)=\sum_{i} \ell_{i j} \cdot e_{i j, a} \cdot e_{i j, b},
$$

where $e_{i j}=\left(e_{i j, 1}, e_{i j, 2}, e_{i j, 3}\right)$ is unit vector in the direction in which the $i$-th ray crosses the $j$-th cell.

By plotting, for each unit vector $e=\left(e_{1}, e_{2}, e_{3}\right)$, the value $\sum_{a, b} R_{a b}(j) \cdot e_{a} \cdot e_{b}$ in the corresponding direction, we get an ellipsoid that describes spatial resolution in different directions. If this ellipsoid is close to a sphere, this means that we have equally good spatial resolution in different directions. If the ellipsoid is strongly tilted in one direction, this means that most of the ray are oriented in this direction, so spatial resolution in this direction is not good.

Limitations of the known approaches. From the application viewpoint, the main limitation is that these methods are, in effect, qualitative, in the following sense:

- the ray coverage, DWS, and the ray density tensor provide us with reasonable indications of where the uncertainty and/or spatial resolution are better and where they are worse;

- however, they do not give a geophysicist any specific guidance on how to use these techniques: what exactly is the accuracy? what exactly is the spatial resolution in different directions?

An additional limitation is that the above methods for gauging uncertainty and spatial resolution are heuristic techniques, they are not justified - statistically or otherwise.

It is therefore desirable to provide justified quantitative estimates for uncertainty and for spatial resolution.

\section{GAuging UnCERTAINTY AND GRANUlARITY (SPATIAl RESOlUtion) OF THE RESUlTS OF SEISMIC Data Processing: New Justifications and NeW TECHNIQUES}

Gauging uncertainty: Gaussian approach. For each cell $j$, each ray $i$ that passes though it leads to an equation $\ell_{i j} \cdot s_{j}+\ldots=t_{i}$. If $\sigma$ is the accuracy with which we measure each traveltime, then, in the assumption that the measurement errors are independent and normally distributed, the probability of a given value $s_{j}$ is proportional to

$$
\prod_{i} \exp \left(-\frac{\left(\ell_{i j} \cdot s_{j}+\ldots-t_{i}\right)^{2}}{2 \sigma^{2}}\right) \text {. }
$$

By using the fact that $\exp (a) \cdot \exp (b)=\exp (a+b)$, we can represent this expression as $\sim \exp \left(-\frac{\left(s_{j}-\ldots\right)^{2}}{2 \sigma(j)^{2}}\right)$, where $(\sigma(j))^{2} \stackrel{\text { def }}{=} \frac{\sigma^{2}}{D_{2}(j)}$, and $D_{2}(j) \stackrel{\text { def }}{=} \sum_{i} \ell_{i j}^{2}$. Thus, the resulting estimate for $s_{j}$ is normally distributed, with standard deviation $\sigma(j)=\frac{\sigma}{\sqrt{D_{2}(j)}}$.

This formula is similar for the formula for the DWS, with the only difference that we add up squares of the lengths instead of the lengths themselves.

Comment: fuzzy approach. We get the same expression if we use a fuzzy approach, with Gaussian membership functions and algebraic product $d \& d^{\prime}=d \cdot d^{\prime}$ as a t-norm.

In practice, we should consider type 2 approaches, i.e., take into account that, e.g., the value $\sigma$ is itself only known with uncertainty - e.g., we know only the interval $[\underline{\sigma}, \bar{\sigma}]$ of possible values of $\sigma$. In this case, we get $\underline{\sigma(j)}=\frac{\underline{\sigma}}{\sqrt{D_{2}(j)}}$ and $\overline{\sigma(j)}=\frac{\bar{\sigma}}{\sqrt{D_{2}(j)}}$

Gauging uncertainty: robust statistical approach. In the case of the normal distribution, finding the most probable values of the parameters is equivalent to the Least Squares method $\sum_{i} \frac{e_{i}^{2}}{\sigma_{i}^{2}} \rightarrow \min$, where $e_{i}$ is the difference between the model and measured values corresponding to the $i$-th measurement. Often, measurement and estimation errors are not normally distributed - and, moreover, we often do not know the shape of the corresponding distribution. In this case, instead of the Least Squares method corresponding to the normal distributions, it makes sense to consider so-called $l^{p}$-methods $\sum_{i} \frac{\left|e_{i}\right|^{p}}{\sigma_{i}^{p}} \rightarrow$ min where a parameter $p$ needs to be empirically determined; see, e.g., [5]. For seismic data processing, the empirical value $p$ is close to 1 ; see [5].

The use of an $l^{p}$-method is equivalent to using the probability distribution $\sim \exp \left(-\frac{\left|e_{i}\right|^{p}}{\sigma^{p}}\right)$. For the seismic case $p=1$ and $e_{i}=\ell_{i j} \cdot s_{j}+\ldots-t_{i}$, we thus get a term proportional to

$$
\prod_{i} \exp \left(-\frac{\left|\ell_{i j} \cdot s_{j}+\ldots-t_{i}\right|}{\sigma}\right) .
$$

By combining the coefficients at $s_{j}$, we thus conclude that the standard deviation is approximately equal to $\sigma(j)=$ $\frac{\sigma}{D(j)}$, where $D(j)=\sum_{i} \ell_{i j}$ is exactly the DWS expression. Thus, in the robust case, we get a statistical justification of the DWS formulas.

Comment. Similar formulas appear if, instead of Gaussian, we use exponential membership functions $\sim \exp (-c \cdot|e|)$. The uncertainty in $\sigma$ can be handled similarly to the Gaussian case.

Gauging spatial resolution. What is the accuracy with which we can determine, e.g., the partial derivative $\frac{\partial s}{\partial x_{1}}$, i.e., in discrete terms, the difference $s^{\prime}-s$, where $s^{\prime} \stackrel{\text { def }}{=}$ $s_{j_{1}+1, j_{2}, j_{3}}$ and $s \stackrel{\text { def }}{=} s_{j_{1}, j_{2}, j_{3}}$ ? This question is best answered 
in the above-described linear approximation. If the ray $i$ is parallel to $x_{1}$ (i.e., $\alpha_{i 1}=0$ ), then, in the formula for $t_{i}$, the values $s$ and $s^{\prime}$ are included with the same coefficient, so we can only determine the average value $\left(s+s^{\prime}\right) / 2$.

In general, the difference between the corresponding interpolation coefficients at $s$ and $s^{\prime}$ is proportional to $\ell_{i j} \cdot \sin \left(\alpha_{i j, 1}\right)$ :

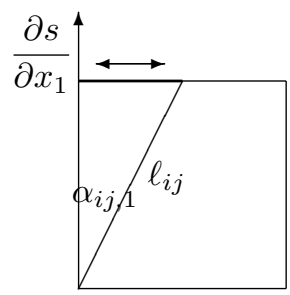

So, in addition to the term proportional to $\left(s+s^{\prime}\right) / 2$, we also get a term proportional to $s^{\prime}-s$, with a coefficient $\ell_{i j} \cdot \sin \left(\alpha_{i j, 1}\right)$. Similarly to the Gaussian approach to uncertainty, we can now argue that the accuracy with which we can determine the desired gradient is proportional to $\sigma_{1}^{2}=\frac{\sigma^{2}}{D_{11}(j)}$, where $D_{11}=\sum_{i} \ell_{i j}^{2} \cdot \sin ^{2}\left(\alpha_{i j, 1}\right)$. In vector terms, $\cos \left(\alpha_{i j, 1}\right)=e_{i j, 1}$, so $\sin ^{2}\left(\alpha_{i j, 1}\right)=1-e_{i j, 1}^{2}$.

Thus, in general, the accuracy in the direction $e=$ $\left(e_{1}, e_{2}, e_{3}\right)$ is $\sim \frac{\sigma}{\sqrt{D_{e}(j)}}$, where $D_{e}(j)=\sum D_{a b}(j) \cdot e_{a} \cdot e_{b}$, and

$$
D_{a b}(j)=D_{2}(j) \cdot \delta_{a b}-\sum_{i} \ell_{i j}^{2} \cdot e_{i j, a} \cdot e_{i j, b}
$$

This formula is similar to the ray density tensor formula, with $\ell_{i j}^{2}$ instead of $\ell_{i j}$. (In the robust case, we get exactly the ray density tensor.)

\section{ACKNOWLEDGMENTS}

This work was supported in part by the National Science Foundation grants HRD-0734825 and DUE-0926721.

\section{REFERENCES}

[1] M. G. Averill, A Lithospheric Investigation of the Southern Rio Grande Rift, PhD Dissertation, University of Texas at El Paso, Department of Geological Sciences, 2007.

[2] M. G. Averill, K. C. Miller, G. R. Keller, V. Kreinovich, R. Araiza, and S. A. Starks, "Using expert knowledge in solving the seismic inverse problem", Int'l J. Approximate Reasoning, 2007, Vol. 45, No. 3, pp. 564-587.

[3] G. Bardossy and J. Fodor, Evaluation of Uncertainties and Risks in Geology, Springer Verlag, Berlin, 2004.

[4] R. Demicco and G. Klir (eds.), Fuzzy Logic in Geology, Academic Press, 2003.

[5] D. I. Doser, K. D. Crain, M. R. Baker, V. Kreinovich, and M. C. Gerstenberger "Estimating uncertainties for geophysical tomography", Reliable Computing, 1998, Vol. 4, No. 3, pp. 241-268.

[6] J. A. Hole, "Nonlinear high-resolution three-dimensional seismic travel time tomography", J. Geophysical Research, 1992, Vol. 97, No. B5, pp. 6553-6562.
[7] E. Kissling, "Geotomography with local earthquake data", Reviews of Geophysics, 1988, Vol. 26, No. 4, pp. 659-698.

[8] E. Kissling, W. Ellsworth, and R. S. Cockerham, "3-D structure of the Long Valley, California, region by tomography", in: D. P. Hill, R. A. Bailey, and A. S. Ryall (eds.), Proc. XIX Workshop on Active Tectonic and Magmatic Processes Beneath Long Valley Caldera, Eastern California, U.S. Geol. Surv., Open-File Report 84-939, 1984, pp. 188-220.

[9] V. Kreinovich and I. Perfilieva, "From gauging accuracy of quantity estimates to gauging accuracy and resolution of measuring physical fields", Proc. 8th Int'l Conf. on Parallel Processing and Applied Mathematics PPAM'2009, Wroclaw, Poland, September 13-16, 2009, to appear.

[10] M. Maceira, S. R. Taylor, C. J. Ammon, X. Yang, and A. A. Velasco, "High-resolution Rayleigh wave slowness tomography of Central Asia", Journal of Geophysical Research, 2005, Vol. 110, paper B06304.

[11] R. L. Parker, Geophysical Inverse Theory, Princeton University Press, Princeton, New Jersey, 1994.

[12] W. Pedrycz, A. Skowron, and V. Kreinovich (eds.), Handbook on Granular Computing, Wiley, Chichester, UK, 2008.

[13] I. Perfilieva and V. Kreinovich, "A broad prospective on fuzzy transforms: from gauging accuracy of quantity estimates to gauging accuracy and resolution of measuring physical fields", Neural Network World, 2010, Vol. 20, No. 1, pp. 7-25.

[14] P. Pinheiro da Silva, A. Velasco, et al., "Propagation and provenance of probabilistic and interval uncertainty in cyberinfrastructure-related data processing and data fusion", In: R. L. Muhanna and R. L. Mullen (eds.), Proc. Int'l Workshop on Reliable Engineering Computing REC'08, Savannah, Georgia, February 20-22, 2008, pp. 199-234.

[15] V. Sallares, J. J. Dañobeitia, and E. R. Flueh, "Seismic tomography with local earthquakes in Costa Rica", Tectonophysics, 2000, Vol. 329, pp. 61-78.

[16] S. Sandoval, E. Kissling, J. Ansorge, and the SVEKALAPKO Seismic Tomography Working Group, "High-resolution body wave tomography beneath the SVEKALAPKO array: II. Anomalous upper mantle structure beneath central Baltic Schield", Geophysical Journal International, 2004, Vol. 157, pp. 200-214.

[17] D. R. Toomey and G. R. Fougler, "Tomographic inversion of local earthquake data from the Hengill-Grensdalur Central Volcano Comple, Iceland", Journal of Geophysical Research, 1989, Vol. 94, No. B12, pp. 17,497-17,510.

[18] G.-A. Tselentis, A. Serpetsidaki, N. Martakis, E. Sokos, P. Paraskevopoulos, and S. Kapotas, "Local high-resolution passive seismic tomography and Kohonen neural networks Application at the Rio-Antirio Strait, central Greece", Geophysics, 2007, Vol. 72, No. 4, pp. B93-B106.

[19] N. Venisti, G. Calcagnile, A. Pontevivo, and G. F. Panza, "Tomographic study of the Adriatic Plate", Pure and Applied Geophysics, 2005, Vol. 162, pp. 311-329.

[20] H. Zhang and C. Thurber, "Development and applications of double-difference seismic tomography", Pure and Applied Geophysics, 2006, Vol. 163, pp. 373-403.

[21] A. Zollo, R. De Matteis, L. D'Auria, and J. Virieux, "A 2D non-linear method for travel time tomography: application to Mt. Vesuvius active seismic data”, In: E. Boschi, G. Ekström, and A. Morelli (eds.), Problems in Geophysics for the New Millennium, Ed. Compositori, Bologna, Italy, 2000, pp. 125140. 